\title{
CONSTRUCTING METRICS WITH THE HEINE-BOREL PROPERTY
}

\author{
ROBERT WILLIAMSON AND LUDVIK JANOS
}

\begin{abstract}
A metric space $(X, d)$ is said to be Heine-Borel if any closed and bounded subset of it is compact. We show that any locally compact and $\sigma$ compact metric space can be made Heine-Borel by a suitable remetrization. Furthermore we prove that if the original metric $d$ is complete, then this can be done so that the new Heine-Borel metric $d^{\prime}$ is locally identical to $d$, i.e., for every $x \in X$ there exists a neighborhood of $x$ on which the two metrics coincide.
\end{abstract}

Introduction. By the Heine-Borel (HB) property of a metric space $(X, d)$ we mean here that every closed bounded set is compact, i.e. bounded sets are totally bounded, and we shall say $d$ is a Heine-Borel metric. ${ }^{1}$ We investigate here how a space can fail to be Heine-Borel. To begin with we are interested in topological conditions that insure that a metrizable space $X$ admits an HB metric $d$. Such a space need not be even finite dimensional. On the other hand it is obvious that any HB space is $\sigma$-compact and locally compact, and we offer in $\S 1$ our first main result, Theorem 1 , as a converse to that.

In $\S 2$ we investigate when a $\sigma$-compact, locally compact metric space $(X, d)$ admits an HB metric which is locally identical to $d$. Note that an HB metric is complete. In Theorem 2 we construct an $\mathrm{HB}$ metric locally identical to a given complete metric. This is not a definitive result, for we construct in Example 1 a metric space which is not complete, yet it too admits a locally identical HB metric. On the other hand, the usual metric $d$ on the open interval $(0,1)$ is an example of a space which does not admit any locally identical metric which is complete (see Remark 2 in §2), and therefore admits no locally identical HB metric.

Finally, in $\S 3$, we present two more examples and we ask: When does a space admit an HB metric which is uniformly locally identical to a given metric? Here a definitive answer is possible, given in Theorem 3. Although the situation is seemingly close to that of $\S 2$ it is in fact simpler. To explore further how a metric space which is $\sigma$-compact, locally compact, and complete can fail to be Heine-Borel we investigate a property common to all our examples of such spaces and close with a conjecture.

Received by the editors June 3, 1985 and, in revised form, December 27, 1985 and May 5, 1986.

1980 Mathematics Subject Classification (1985 Revision). Primary 54D45, 54E35, 54E50.

Key words and phrases. Heine-Borel property, uniformly locally compact metric space, paracompact space.

${ }^{1}$ Some of these results were announced in Abstracts of the American Mathematical Society \#816-99-520 by Williamson and Janos in January 1985 and \#85T-54-168 by Williamson in June 1985 ; in both of these the term quasi-Euclidean was used instead of Heine-Borel. 
1. Construction of a Heine-Borel metric. The following theorem is due to Vaughan [7] and appears in [2] with a proof similar to the one we give (see also [4, problem $4.2 \mathrm{C}]$ ).

THEOREM 1. If $X$ is a $\sigma$-compact, locally compact, metrizable space, then there is a compatible metric on $X$ which is Heine-Borel.

ProOF. We say a subset of $X$ is precompact if it has compact closure. Since $X$ is $\sigma$-compact and locally compact, $X$ can be represented as the union of an increasing sequence (finite or infinite) of open precompact sets $X_{n}$ such that the closure $\mathrm{Cl}\left(X_{n}\right)$ is properly contained in $X_{n+1}$ (see e.g. [4 or 9]). We shall call such a sequence an exhaustion of $X$.

Using the normality of $X$ we construct continuous functions $f_{n}: X \rightarrow[0,1]$ such that $f_{n}(x)$ is 0 for $x \in X_{n}$ and is 1 for $x \in X-X_{n+1}$. Observing that the function

$$
f(x)=\sum_{n=1}^{\infty} f_{n}(x)
$$

is well defined and continuous, we define

$$
d^{\prime}(x, y)=d(x, y)+|f(y)-f(x)|
$$

where $d$ is an arbitrary compatible metric on $X$. We observe that $d^{\prime}$ is a metric on $X$. In order to show that $d^{\prime}$ is equivalent to $d$, we first observe that $d^{\prime} \geq d$ so we only need to show that a sequence $\left\{x_{n}\right\}$ converges to $x$ relative to $d^{\prime}$ if it converges relative to $d$, and this follows from the continuity of $f$. Now any $d^{\prime}$-bounded set $A$ is contained in some $X_{n}$, by the definition of $f$, so it has compact closure. Thus $d^{\prime}$ is a Heine-Borel metric.

2. Locally identical metrics. We say two metrics $d$ and $d^{\prime}$ on $X$ are locally identical iff every point $x \in X$ has a neighborhood $V$ such that $d(z, y)=d^{\prime}(z, y)$ for $z, y \in V$. Equivalently, there is a neighborhood of the diagonal in $X \times X$ on which $d$ and $d^{\prime}$ coincide.

On $R^{n}$ (or any unbounded HB space $(X, d)$ ) one can construct an equivalent bounded metric $d^{*}$ by setting $d^{*}(x, y)=\min \{d(x, y), 1\}$, and this is complete but not HB. Is this example in any sense typical, and if so, how does one reconstruct an HB metric from it? The rest of this note is an investigation of this question. Similar examples suggest that the essential thing is that the bounded metric is locally identical to the given metric. Our second main resuslt is

THEOREM 2. A metric space $(X, d)$ has a Heine-Borel metric which is locally identical to $d$ if it is complete, $\sigma$-compact, and locally compact.

Example 1 shows that an incomplete metric space may also admit a locally identical HB metric, but we can put the result above in the more definitive form of Theorem $2^{\prime}$ as follows. We shall say that metrics $d$ and $d^{\prime}$ are Cauchy equivalent when a sequence is Cauchy relative to $d$ if and only if it is Cauchy relative to $d^{\prime}-$ note that this does not imply uniform equivalence (see Example 2). We shall say metrics $d, d^{\prime}$ which are Cauchy equivalent and locally identical are Cauchy locally identical. 
THEOREM 2'. A metric space $(X, d)$ has a Heine-Borel metric which is Cauchy locally identical to $d$ if and only if it is complete, $\sigma$-compact, and locally compact.

It is easily seen that two complete equivalent metrics are Cauchy equivalent, so this follows immediately from Theorem 2 .

PROOF OF THEOREM 2. We shall sometimes call a finite sequence of points in $X,\left\langle x_{i} \mid i=1, \ldots, n\right\rangle$, a chain (from $x_{1}$ to $x_{n}$ ), and define the length of the chain to be $\sum d\left(x_{i}, x_{i+1}\right)$.

Let $W$ be a covering of the metric space $(X, d)$. We define a relation on $X$, also denoted by $W$, by setting $x W y$ iff there is an $A \in W$ such that $x, y \in A$, and we define $x \equiv_{W} y$ (or just $x \equiv y$ ) if there is a chain $\left\langle x_{i} \mid i=1, \ldots, n\right\rangle$ from $x$ to $y$ such that $x_{i} W x_{i+1}$ for all $i<n$. We say $\left\langle x^{n}\right\rangle$ is a $W$-chain and $x$ and $y$ are $W$-connected; this is evidently an equivalence relation, and we call the equivalence classes $W$-chain classes. On any equivalence class of $\equiv$ we define the $W$-chain metric $d_{W}$ by

DEFINITION. For $x \equiv y, d_{W}(x, y)$ is the infimum of the lengths of all $W$-chains from $x$ to $y$.

We henceforth asume that for any $x, y \in X$ there is a $W$-chain from $x$ to $y$, so there is only one equivalence class; we then say $X$ is $W$-chain connected. Of course if $X$ is connected it is $W$-chain connected for any open covering $W$.

For a metric space $X$ with covering $W$, the chain metric $d_{W}$ is easily seen to be a metric, and it is locally identical to the original metric. It is evident that the chain metric $d_{W}$ is the largest metric which agrees with $d$ on every set of $W$.

If $x_{1}, \ldots, x_{n}$ is a $W$-chain and $x_{i} W x_{j}$ for some $j>i$, one can delete all the points between $x_{i}$ and $x_{j}$ to produce a chain with fewer points and length no greater than the original. This process can be repeated until, for $i<j, x_{i} W x_{j}$ iff $j=i+1$; we call such a chain a reduced chain.

It is evident that Theorem 2 follows from the following proposition, in which a Heine-Borel metric $d_{V}$ is constructed.

PROPOSITION. Let $X_{1}, X_{2}, \ldots$ be an exhaustion (in the sense of $\S 1$ ) of the complete, locally compact metric space $(X, d)$. Let $V_{n}=X_{n}-\mathrm{Cl}\left(X_{n-2}\right)$, with the convention that $X_{n}=\varnothing$ for $n \leq 0$. Let $V=\left\{V_{n} \mid n=2,3, \ldots\right\}$. Then any $d_{V}$-bounded set is precompact.

PROOF. By the height of $x, h(x)$, we mean the smallest $n$ such that $x \in X_{n}$. If $x_{1}, \ldots, x_{n}$ is a $V$-reduced chain and $h\left(x_{1}\right)<h\left(x_{n}\right)$, then for each integer from $h\left(x_{1}\right)$ to $h\left(x_{n}\right)$ there will be exactly one point of the chain with that integer as height; this follows from the definition of the $V_{n}$. Thus when $h\left(x_{1}\right)=1$, we have $h\left(x_{i}\right)=i$ for $i=1, \ldots, k$. Recall that we insist that $\mathrm{Cl}\left(X_{n}\right)$ is properly contained in $X_{n+1}$, and this implies that $X$ is $V$-connected.

Now suppose the proposition is false. Then there is a bound $K$ and a sequence of points $\left\langle x_{n}\right\rangle$ with no convergent subsequence such that $d_{V}\left(x_{1}, x_{n}\right)<K$ for all $n$, and we take $x_{1}$ to be in $X_{1}$. So each $x_{i}$ admits a $V$-chain $x_{i, 1}, \ldots, x_{i, n(i)}$ from $x_{1}$ to $x_{i}$ of length less than $K$, and we may as well suppose it is reduced, so $h\left(x_{i, k}\right)=k$. Then $x_{1, i}$ is $x_{1}$ for all $i$; let $y_{1}$ be this point. The sequence of points $x_{i, 2}$ in $X_{2}$, which is precompact, must have a subsequence, with indices $i=i_{2}(j)$ say, converging to some point $y_{2}$. The corresponding subsequence of the $x_{i, 3}$ must in turn have a subsequence with indices $i_{2} i_{3}(j)$ say (where $i_{2} i_{3}$ is the composition 
of the functions), converging to some point $y_{3}$. We proceed in this way inductively, selecting $y_{n}$ for every $n$. Now we show the

LEMMA. The length of the chain $y_{1}, \ldots, y_{n}$ is no greater than $K$ for every $n$.

PROOF OF THE LEMMA. By construction of the $y_{i}$ there is an indexing $i=$ $i_{1} i_{2} \cdots i_{n}(j)$ such that for each $k=1, \ldots, n$ the corresponding subsequence $\left\langle x_{i(j), k}\right\rangle$ converges to $y_{k}$. Thus for any $\delta>0$ there is an $N$ such that for all $j>N$ and $k=1, \ldots, n, d\left(x_{i(j), k}, y_{k}\right)<\delta / n$. So for $j>N$ we have, for $i=i(j)$ and with $k$ ranging from 1 to $n-1$ in the sums,

$$
\begin{aligned}
\sum d\left(y_{k}, y_{k+1}\right) & \leq \sum d\left(y_{k}, x_{i, k}\right)+\sum d\left(x_{i, k}, x_{i, k+1}\right)+\sum d\left(x_{i, k+1}, y_{k+1}\right) \\
& \leq 2 \delta+K
\end{aligned}
$$

Since this holds for all $\delta$, the Lemma is proved.

This shows that $\sum d\left(y_{k}, y_{k+1}\right)$ is convergent, so $y_{n}$ is a Cauchy sequence relative to the original metric $d$. Since $X$ is complete this sequence converges to a point $y$, say. But where could $y$ be? It must be in some $X_{N}$, but these are open, so we would then have $y_{n} \in X_{N}$ for all sufficiently large $n$. But $y_{n}$ is the limit of points in $V_{n}$, hence in the complement of $X_{n-2}$, which is closed, so $y_{n}$ is also in the complement of $X_{n-2}$. Since this holds for every $n$ we reach a contradiction which establishes the Proposition. Note that we make no assertion about the convergence of any subsequence of the original $x_{n}$. This completes the proof of Theorem 2 .

The chain metric of a covering does not seem to be in the literature in quite this form, although similar ideas have been often used; for example in $[\mathbf{1}, \mathbf{3}, \mathbf{5}, \mathbf{6}]$.

We present an example. To describe this and the following examples it will be convenient to generalize the chain construction slightly. Suppose $X$ is an abstract set covered by a family $W$ of subsets which are metric spaces such that $W$ is coherent, in the sense that if $(A, d)$ and $\left(B, d^{\prime}\right)$ are in $W$, then $d$ and $d^{\prime}$ agree on $A \cap B$. Then we can say what it means for $X$ to be $W$-chain connected and we can define the chain metric $d_{W}$ exactly as before. Again, it will be the largest metric which agrees with $d$ on $A$ for any $(A, d) \in W$. The properties of the examples are easily verified by elementary arguments. By $N$ we mean the set of positive integers, and we will let $n$ be a variable over $N$ and $x, y$ variables over $R$.

EXAMPLE 1. Let $W=\left\{K_{n} \mid n \in N\right\} \cup\left\{H_{n} \mid n \in N\right\}$, where $K_{n}=\{n\} \times R$ has metric $k_{n}((n, x),(n, y))=\min \left\{|x-y|, 2^{-n}\right\}$ and $H_{n}=[n, n+1] \times\{n\}$ has metric $h_{n}((x, n),(y, n))=\left(1 / 2^{n}\right)|x-y|$. Let $X_{1}=\bigcup W$ with the chain metric $d_{1}=d_{W}$. This space is not complete since the sequence $(n, n)$ does not converge. Nevertheless for a suitable exhaustion the chain metric does produce a metric which is locally identical, but not Cauchy equivalent, to $d_{1}$. So the question remains open: What are necessary conditions on a metric space so that it admits a locally identical Heine-Borel metric?

REMARK 1. If the open unit interval $(0,1)$ is given the usual metric $d$, it is easily seen that the chain metric for any covering $W$ coincides with $d$. Since, as noted above, the chain metric is the largest metric that coincides with $d$ on each set of the covering, it is evident that any metric locally identical to $d$ cannot exceed $d$, and therefore cannot be Heine-Borel.

3. Uniformly locally identical metrics. Recall that metrics are uniformly equivalent if they generate the same uniform structure, i.e. the same surroundings 
of the diagonal in $X \times X$. We say metrics $d, d^{\prime}$ on $X$ are uniformly locally identical if they are uniformly equivalent and coincide on some surrounding of the diagonal. A metric space is uniformly locally compact (see [9]) if there is a $\delta>0$ such that any open ball of radius $\delta$ is precompact. Consider the following example, which is constructed using the method of Example 1 above:

EXAMPLE 2. Let $W=\left\{L_{n} \mid n \in N\right\} \cup\left\{H_{n} \mid n \in N\right\}$ where $L_{n}=\{n\} \times R$ with metric $e_{n}((n, x),(n, y))=\min (|x-y|, 1 / n)$, and $H_{n}=[n, n+1] \times\{0\}$ with the usual metric. Let $X_{2}=\bigcup W$ with the metric $d_{2}=d_{W}$.

The space $X_{2}$ is complete and the chain construction of Theorem 2 supplies an HB metric $d_{2}^{\prime}$ which is Cauchy locally identical to $d_{2}$. Indeed, for a suitable covering, $d_{2}^{\prime}$ is the same as $d_{2}$ with $e_{n}$ replaced by $e_{n}^{\prime}$ in the construction, where $e_{n}^{\prime}((n, x),(n, y))=|x-y|$. Nevertheless, since $\left(X_{2}, d_{2}\right)$ is not uniformly locally compact, there is no HB metric which is uniformly equivalent to $d_{2}$, so certainly none which is uniformly locally identical to $d_{2}$. This incidentally provides a simple example of metrics that are Cauchy equivalent but not uniformly equivalent. This distinction will be important in what follows, for in the context of uniform local identity a definitive result is possible, and is in fact less delicate than Theorem 2.

THEOREM 3. A metric space $(X, d)$ has a Heine-Borel metric $d^{\prime}$ which is uniformly locally identical to $d$ if and only if $(X, d)$ is $\sigma$-compact and uniformly locally compact.

PROOF. We first note that a uniformly locally compact space is automatically complete. Now the "only if" part follows from the observation that any HB metric is uniformly locally compact, and the property is certainly preserved under uniform local identity.

The proof of Theorem 2 will give us the "if" part of the theorem as well if we can choose the exhaustion used there so that the cover $V$ constructed from it is a uniform cover. That is the content of the next lemma.

Let $B[\delta]$ be the covering consisting of all open balls $B(x, \delta)$ of radius $\delta$ and center $x$ for any $x \in X$.

LEMMA. Suppose every open $2 \delta$-ball is precompact. Then there is a locally finite cover $W$ of precompact open sets such that $B[\delta]$ refines $W$ (which is therefore uniform) and $X$ is $W$-chain connected. There corresponds to $W$ an exhaustion $\left\langle X_{n}\right\rangle$ of $X$ such that the associated cover $V=\left\{V_{n} \mid n \in N\right\}$, where $V_{n}=X_{n}-\mathrm{Cl}\left(X_{n-2}\right)$, is refined by $W$.

ProOF. We will construct the cover $W$ and an exhaustion at the same time. Choose some $x \in X$ and let $W_{1}=\{B(x, 2 \delta)\}, X_{1}=B(x, 2 \delta)$. For the purpose of induction suppose that the sequence of partial coverings $W_{1}, \ldots, W_{n}$, has been constructed where each $W_{k}$ is a finite collection of open precompact sets, and $W_{k}$ includes $W_{k-1}$. Let $X_{k}=\bigcup\left\{A \mid A \in W_{k}\right\}$. Further, suppose that

(i) $d\left(X_{k}^{c}, X_{k-1}\right) \geq \delta$ for $k \leq n$,

(ii) none of the sets in $W_{k}-W_{k-1}$ meet $X_{k-2}$,

(iii) for any $x \in X_{n-1}, B(x, \delta)$ is contained in some element of $W_{n}$.

Then cover $\mathrm{Cl}\left(X_{n}\right)$ by $\delta$-balls, and select a finite subcover, based at $x_{1}, \ldots, x_{s}$, say. Let $B_{i}=B\left(x_{i}, 2 \delta\right) \cap \mathrm{Cl}\left(X_{n-2}\right)^{c}$. Set $W_{n+1}=W_{n} \cup\left\{B_{1}, \ldots, B_{s}\right\}$, but discard the empty $B_{i}$ 's and define $X_{n+1}$ as above. Then $W_{n+1}$ is a cover of $X_{n+1}$ by open 
precompact sets. It follows easily that (i) and (ii) hold for $k+1$. Finally, suppose $x \in X_{n}-X_{n-1}$. Then there is an $x_{i}$ with $d\left(x, x_{i}\right) \leq \delta$, so $B(x, \delta)$ is contained in $B\left(x_{i}, 2 \delta\right)$; since $d\left(x, X_{n-2}\right)>\delta$ by (i), $B(x, \delta)$ is contained in $X_{n-2}^{c}$ as well, so $B(x, \delta)$ is contained in $B_{i}$. Since (iii) already holds for $x \in X_{n-1}$, this verifies it for $n+1$ as well.

Assume for the moment that $X$ is $B[\delta]$-chainable. Then for any $x \in X$ there is a chain $B_{1}, \ldots, B_{q}$ of $\delta$-balls from $x_{1}$ to $x$, and (i) guarantees that $B_{i}$ is contained in $X_{i}$, by an easy induction. Thus $\bigcup X_{n}=X$. It is then obvious from (i) that the $X_{n}$ form an exhaustion, at least if $X$ is not compact; if it is, the argument is trivial. Evidently $\bigcup W_{n}$ covers $X$, and (ii) guarantees that the covering is locally finite, while (iii) says it is refined by $B[\delta]$.

Finally, if $X$ is not $B[\delta]$-chain connected, we proceed as follows. We enumerate the equivalence classes as $E_{1}, \ldots$ and select a $\delta$-ball $B_{i}$ contained in $E_{i}$. Then we adjoin $B_{i}$ to $W_{i}$. This does not affect the construction above, and completes the proof of Theorem 3.

We remark that the proof of Theorem 2 is not really needed. We can just use property (i) in the proof of the Lemma to show that if a set $A$ is $d_{V}$-bounded by $K$ and $N \delta>K$, then $A$ is contained in $X_{N}$. It does not seem that the method used here can be generalized to prove Theorem 2 .

EXAMPLE 3. In this example $n$ ranges over the positive integers $N$. Let $K_{n}=$ $\{(x, y) \mid x=n$ and $0 \leq y \leq 1 / n\} \cup\{(1,1)\}, H_{n}=\{(x, 0) \mid n \leq x \leq n+1\}$, and $N^{\prime}=\{(n, 0) \mid n=1,2, \ldots\}$. Let $W=\left\{K_{n} \mid n \in N\right\} \cup\left\{H_{n} \mid n \in N\right\} \cup\left\{N^{\prime}\right\}$. Projection on the second factor embeds $K_{n}$ in $[0,1]$, and we give $K_{n}$ the induced metric. Let $H_{n}$ have the usual metric and let $N^{\prime}$ have the discrete metric of diameter 1, i.e. the distance between any two points is 1 . Then we set $X_{3}=\bigcup W$ and $d_{3}=d_{W}$. One can regard $X_{3}$ as a subspace of the complete graph on the points $N^{\prime} \cup\{(1,1)\}$.

The space $X_{3}$ is uniformly locally compact. Although $d_{3}$ is not HB, by Theorem 3 there is an HB metric uniformy locally identical to $d_{3}$. The space $X_{3}$ has the property that $B_{r}=B((1,1), r)$ is precompact if $r<1$ but the closure of $B_{1}$, which is $\bigcup L_{n}$, is not compact - this does not occur in the "bounded metric" model $d^{*}$ of $\S 2$. We introduce some notation to pursue this a little further.

Let $B(x, r)$ be the open ball of radius $r$ and center $x$ and let $C B(x, r)=$ $\{z \mid d(x, z) \leq r\}$ be the closed ball, so $\mathrm{Cl}(B(x, r))$ is contained in $C B(x, r)$. The second remark has to do with the relation between the HB property and the difference between these two subsets. A bounded metric $d^{*}$, in the sense of $\S 1$, has several properties that seem to be related to the failure to be HB. For any space $X$ which is complete, $\sigma$-compact, and locally compact, let $r_{x}=\sup \{r \mid B(x, r)$ is precompact $\}$ - we will call $r_{x}$ the compact radius of $X$ - so $X$ is HB iff $r_{x}=\infty$ for some $x$. If $r_{x}$ is finite one can show $C B\left(x, r_{x}\right)$ cannot be compact. On the other hand, for the bounded metric $d^{*}$ of $\S 2, \mathrm{Cl}\left(B\left(x, r_{x}\right)\right)$ is compact. Furthermore in the case of $\left(R, d^{*}\right)$ the chain metric could be based on the cover of all precompact balls, without the condition of local finiteness, so that on every precompact ball the original metric coincides with the $\mathrm{HB}$ chain metric. But there is a complete $\sigma$-compact, locally compact space $X$ with $x \in X$ such that $\operatorname{Cl}\left(B\left(x, r_{x}\right)\right)=C B\left(x, r_{x}\right)$. Such an example, incidentally, cannot be uniformly locally compact. It follows that any HB metric on $X$ (which exists by the theorem) cannot agree with the original metric on the whole open ball $B\left(x, r_{x}\right)$, and it follows that the cover of all precompact balls 
cannot be used in the chain construction; some condition such as local finiteness is called for. We do find one feature common to all our examples of spaces which fail to be Heine-Borel, so we make the following

CONJECTURE. A complete admissible metric for a $\sigma$-compact, locally compact space $X$ is always a Heine-Borel metric if $\mathrm{Cl}(B(x, r))=C B(x, r)$ for every $x \in X$ and $r>0$.

\section{REFERENCES}

1. Leonard M. Blumenthal, Theory and application of distance geometry, Chelsea, New York, 1970.

2. H. Busemann, Local metric geometry, Trans. Amer. Math. Soc. 56 (1944), 200-274.

3. Michael Edelstein, An extension of Banach's contraction mapping principle, Proc. Amer. Math. Soc. 12 (1961), 7-10.

4. R. Engelking, General topology, Polish Scientific Publishers, Warsaw, 1977.

5. Julien Garsoux, Espaces vectoriels topologiques et theorie des distributions, Dunod, Paris, 1963.

6. A. A. Ivanov, Fixed points of selfmaps on metric spaces, Zap. Nauchn. Sem. Leningrad. Otdel. Mat. Inst. Steklov. (LOMI) 66 (1976), 5-102.

7. H. E. Vaughan, On locally compact metrizable spaces, Bull. Amer. Math. Soc. 43 (1937), 532535 .

8. Stephen Willard, General topology, Addison-Wesley, Reading, Mass., 1970.

Department of Mathematics, Claremont Graduate School, Claremont, CALIFORNIA 91711 